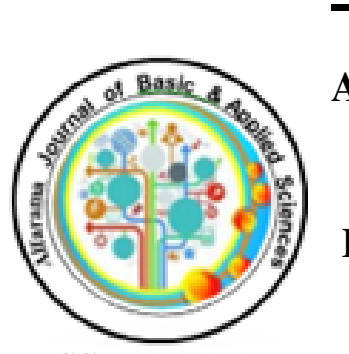

ISSN 2682-275X
Alfarama Journal of Basic \& Applied Sciences

https://ajbas.journals.ekb.eg/

ajbas@sci.psu.edu.eg

Faculty of Science Port Said University

http://sci.psu.edu.eg/en/

January 2021, Volume 2, Issue 1 DOI: 10.21608/ajbas.2020.39888.1030

Submitted: $21-08-2020$

Accepted: 16-09-2020

Pages: 135-148

\title{
Modulation of the Nonlinear Ion Acoustic Waves in a Weakly Relativistic Warm \\ Plasma with Nonextensively Distributed Electrons
}

\author{
Salah K. El-Labany ${ }^{1,2}$, Wael F. El-Taibany ${ }^{1,2}$, Nabila A. El-Bedwehy ${ }^{2,3}$, Nora A. El-Shafeay ${ }^{1, *}$ \\ ${ }^{1}$ Department of Physics, Faculty of Science, Damietta University, New Damietta, P.O. 34517, Egypt \\ ${ }^{2}$ Center of Space Research and its Applications (CSRA), Damietta University, New Damietta, P.O. 34517, Egypt \\ ${ }^{3}$ Department of Mathematics, Faculty of Science, Damietta University, New Damietta, P.O. 34517, Egypt \\ *Corresponding author: naelshafeay@gmail.com
}

\begin{abstract}
A reductive perturbation technique (multiple scales) is applied to a weakly relativistic warm unmagnetized adiabatic plasma system consisting of inertial ions fluid and nonextensively distributed electrons. A nonlinear Schrödinger-type (NST) equation for finite wavenumber at the second order is derived. Using the reductive perturbation technique we derived the corresponding Korteweg-de Vries (K-dV) equation. For small wavenumber limit the K-dV equation is transformed into NST equation. It is found that the coefficient of the NST equation obtained from the $\mathrm{K}-\mathrm{dV}$ equation agree with the corresponding coefficients of NST equation obtained by the multiple scales. Moreover we investigated the effect of the physical parameters of the system namely temperature ratio of the ion temperature $T_{i}$ to electron temperature $T_{e}$, the relativistic factor $u_{0} / C$ as well as the nonextensive parameter $(q)$ of the distribution on the stability/instability of the system. It is found that these parameters affect strongly on the stability/instability regions. Finally, the validity of our results in astrophysical plasma is briefly discussed.
\end{abstract}

\section{Keywords}

Plasma Physics, Modulation Instability, Weakly Relativistic, Nonextensive Electrons.

\section{INTRODUCTION}

Ion acoustic wave (IAW) is one type of longitudinal oscillations of ions and electrons in plasma systems. It is similar to the acoustic waves traveling in neutral gases. The propagation of nonlinear IAWs in a weakly dispersive medium has been investigated theoretically and experimentally[1, 2, 3, 
4, 5]. Ikezi et al. [6] have reported the first experimental observation of IAWs solitons. Watanabe [7] studied the modulation instability of the monochromatic IAWs experimentally. The modulation instability of IAWs in warm non-relativistic plasma has been studied by Xue et al.[8].

The nonextensive statistic mechanics, based on the deviations from Boltzmann-Gibbs-Shannon (BGS) statistics has been studied in the last few decades. A suitable nonextensive generalization of the BGS entropy for statistical equilibrium was first investigated by Renyi [9] and afterwards suggested by Tsallis[10]. Tsallis extended the standard additivety of the entropies of the nonlinear systems. This nonadditive entropy of Tsallis and the generalized statistics have been investigated in different phenomena characterized by nonextensivity $[11,12,13,14,15,16,17,18,19]$ through the entropic index $q$, characterizes the degree of nonextensivity of the considered system while the standard extensive BGS statistics is at $q=1$. The nonextensive statistics are successfully applied to many astrophysical scenarios such as stellar polytropes, solar neutrino problem, and peculiar velocity distribution of galaxy clusters[20, 21].

If the particle velocity is much less than the velocity of light, ion waves will exhibit non-relativistic behavior, but when the particle velocity approaches that of light, relativistic effect must be considered. The modulation instability of IAWs in a weakly relativistic warm plasma for different distribution has been studied by El-Labany [22] and El-Labany et al.[23, 24]. The cold nonrelativistic modulation instability has been studied for different distributions. It has been studied using nonthermal distribution by Zhang et al.[25], q-nonextensive distribution by Bains et al. [21], and superthermal (kappa) distribution by Guo and Mei [26] and Chowdhury et al. [27]. The nonlinear evolutions in plasmas are investigated by different approximation techniques, in which one assumes small deviations for system from the equilibrium state of the linear wave. In fact such multiple scales method [23, 24], the reductive perturbation technique (RPT) [21] and Krylov-Bogoliubov-Mitropolsky method (KBM) [28] which lead to nonlinear Schrödinger-type (NST) equation. However, the system of a weakly relativistic warm unmagnetized adiabatic plasma consisting of inertial ions fluid and nonextensively distributed electrons has not been investigated; this is our goal.

The skeleton of this article is as follow:

In section 2 we present the basic system of equations representing our model and we derive the NST equation. In section 3 we derive the small wavenumber approximation Korteweg-de Vries (K-dV) equation. In section 4 we transform the K-dV equation obtained in section 3 to the NST equation and results and discussion in section 5. Section 6 is devoted conclusion.

\section{BASIC EQUATIONS AND DERIVATION OF THE NST EQUATION}

Consider a simple model of adiabatic unmagnetized collisionless weakly relativistic plasma that contains one warm ion species together with nonextensively distributed electrons. The one-dimensional basic equations can be written in non-dimensional form as

$$
\begin{gathered}
\frac{\partial n}{\partial t}+\frac{\partial(n u)}{\partial x}=0 \\
\left(\frac{\partial}{\partial t}+u \frac{\partial}{\partial x}\right)(\gamma u)+3 \sigma n \frac{\partial n}{\partial x}+\frac{\partial \Phi}{\partial x}=0, \\
\frac{\partial^{2} \Phi}{\partial x^{2}}=n_{e}-n, \\
n_{e}=[1+(q-1) \Phi]^{\frac{(q+1)}{(q-1)}} \approx 1+\alpha_{1} \varphi-\alpha_{2} \varphi^{2}+\alpha_{3} \varphi^{3}+\ldots,
\end{gathered}
$$


where,

$$
\left.\begin{array}{c}
\alpha_{1}=\frac{q+1}{2}, \\
\alpha_{2}=\frac{(q+1)(q-3)}{8}, \\
\alpha_{3}=\frac{(q+1)(q-3)(3 q-5)}{48} .
\end{array}\right\}
$$

and $n, n_{e}$ are the densities of the ions and electrons respectively, $\mathrm{u}$ is the flow velocity of the ions, $\Phi$ is the electrostatic potential, $x$ is the space coordinate, $\mathrm{t}$ is the time variable, $\sigma \ll 1$ is the ratio of ion temperature $T_{i}$ to electron temperature $T_{e}$ and the parameter q stands for the strength of nonextensively, and $\gamma$ is the relativistic factor

$$
\gamma=\left(1-\frac{u^{2}}{C^{2}}\right)^{\frac{-1}{2}}
$$

Assuming a weakly relativistic effect the relativistic factor can be approximated by its expansion up to the second term .i.e. [29]

$$
\gamma \approx 1+\frac{u^{2}}{2 C^{2}}
$$

All physical quantities in Eqs. (1)-(4), $u, \Phi, n, x$ and $t$ are normalized with respect to thermal velocity $\left(k_{B} T_{e} / m\right)^{(1 / 2)}$, thermal potential $\left(k_{B} T_{e} / e\right)$, unperturbed ion density $n_{0}$, Debye length $\lambda_{D}=$ $\left(k_{B} T_{e} / 4 \pi e^{2} n_{0}\right)^{(1 / 2)}$ and the inverse of the ion plasma frequency $\omega_{p i}^{-1}=\left(4 \pi e^{2} n_{0} / m\right)^{(1 / 2)}$ respectively, where $\mathrm{m}$ is the ion mass, $k_{B}$ is the Boltzmann constant and $\mathrm{e}$ is the electron charge. To derive the nonlinear Schrödinger-type equation, we employ the general method of a multiple scales. In this method we introduce the independent variables [22]

$$
\tau_{i}=\varepsilon^{i} t, \quad \xi_{0}=x, \text { and } \xi_{i}=\varepsilon^{i}(x-\lambda t)(i=1,2, \ldots) .
$$

Thus the time and space derivatives in Eqs. (1-4) can be written as [24]

$$
\left.\begin{array}{c}
\frac{\partial}{\partial t} \longrightarrow \frac{\partial}{\partial \tau_{0}}+\varepsilon\left(\frac{\partial}{\partial \tau_{1}}-\lambda \frac{\partial}{\partial \xi_{1}}\right)+\varepsilon^{2}\left(\frac{\partial}{\partial \tau_{2}}-\lambda \frac{\partial}{\partial \xi_{2}}\right)+\ldots, \\
\frac{\partial}{\partial x} \longrightarrow \frac{\partial}{\partial \xi_{0}}+\varepsilon \frac{\partial}{\partial \xi_{1}}+\varepsilon^{2} \frac{\partial}{\partial \xi_{2}}+\ldots,
\end{array}\right\}
$$

where $\varepsilon$ is a small dimensionless parameter representing the size of the perturbed amplitude and $\lambda$ represented the group velocity $\left(\lambda=\frac{\partial \omega}{\partial k}\right)$; will be determined later. Now we expand the variables $n, u$, $\Phi$ in terms of the expansion parameter $\varepsilon$ as (EL-Labany 1995 [22])

$$
\begin{aligned}
& n=1+\sum_{m=1}^{\infty} \varepsilon^{m} \sum_{l=-m}^{m} n_{m}^{(l)}\left(\tau_{1}, \tau_{2}, \ldots, \xi_{1}, \xi_{2}, \ldots\right) \exp [i l(k x-\omega t)], \\
& \left.u=u_{0}+\sum_{m=1}^{\infty} \varepsilon^{m} \sum_{l=-m}^{m} u_{m}^{(l)}\left(\tau_{1}, \tau_{2}, \ldots, \xi_{1}, \xi_{2}, \ldots\right) \exp [i l(k x-\omega t)],\right\} \\
& \Phi=\sum_{m=1}^{\infty} \varepsilon^{m} \sum_{l=-m}^{m} \Phi_{m}^{(l)}\left(\tau_{1}, \tau_{2}, \ldots, \xi_{1}, \xi_{2}, \ldots\right) \exp [i l(k x-\omega t)],
\end{aligned}
$$

where $n, u$ and $\Phi$ are satisfied the reality condition $A_{-l}^{(m)}=A_{l}^{(m) *}$ and the asterisk denotes the complex conjugate. Substituting Eqs. (7) into the basic equations (1-4), we obtain to the first order of $\varepsilon$ and 
$l=1$

$$
\left.\begin{array}{c}
u_{1}^{(1)}=\frac{\tilde{\omega}}{k} n_{1}^{(1)}, \\
\text { and } \\
\Phi_{1}^{(1)}=\frac{n_{1}^{(1)}}{\left(k^{2}+\alpha_{1}\right)} .
\end{array}\right\}
$$

With the linear dispersion relation and group velocity $\lambda$ given respectively by

$$
\tilde{\omega}^{2} \gamma_{1}=3 \sigma k^{2}+\frac{k^{2}}{\left(k^{2}+\alpha_{1}\right)},
$$

and

$$
\lambda=u_{0}+\frac{k}{\gamma_{1} \tilde{\omega}}\left(3 \sigma+\frac{\alpha_{1}}{\left(k^{2}+\alpha_{1}\right)^{2}}\right) .
$$

where $\gamma_{1}=1+\frac{3 u_{0}^{2}}{2 C^{2}}$ and $\tilde{\omega}=\omega-k u_{0}$.

The components of $\mathrm{O}(\varepsilon)$ for $l=0$ are given by,

$$
\left.\begin{array}{c}
n_{1}^{(0)}=n_{e 1}^{(0)}, \\
\text { and } \\
\Phi_{1}^{(0)}=\frac{n_{1}^{(0)}}{\alpha_{1}},
\end{array}\right\}
$$

However the second order harmonic terms $\mathrm{O}\left(\varepsilon^{2}\right)$ of the reduced equations, with $l=0$ are given by,

$$
\left.\begin{array}{c}
\frac{\partial n_{1}^{(0)}}{\partial \xi_{1}}=\frac{\partial u_{1}^{(0)}}{\partial \xi_{1}}=\frac{\partial \Phi_{1}^{(0)}}{\partial \xi_{1}}=0, \\
\Phi_{1}^{(0)}=0, \\
\Phi_{2}^{(0)}=\frac{\left(n_{2}^{(0)}-2 \alpha_{2}\left|\Phi_{1}^{(1)}\right|^{2}\right)}{\alpha_{1}},
\end{array}\right\}
$$

provided that

$$
\gamma_{1} \tilde{\lambda}^{2} \neq \frac{1}{\alpha}{ }_{1}+3 \sigma
$$

For $l=1$ components,

$$
\left.\begin{array}{c}
\frac{\partial n_{1}^{(1)}}{\partial \tau_{1}}=0, \\
u_{2}^{(1)}=\frac{\tilde{\omega}}{k} n_{2}^{(1)}+\frac{i}{k}\left(\frac{\tilde{\omega}}{k}-\tilde{\lambda}\right) \frac{\partial n_{1}^{(1)}}{\partial \xi_{1}}, \\
\Phi_{2}^{(1)}=\frac{n_{2}^{(1)}}{\left(k^{2}+\alpha_{1}\right)}+\frac{2 i k}{\left(k^{2}+\alpha_{1}\right)^{2}} \frac{\partial n_{1}^{(1)}}{\partial \xi_{1}} ;
\end{array}\right\}
$$

i.e. no $\tau_{1}$ dependent.

For $l=2$ components,

$$
\left[n_{2}^{(2)}, u_{2}^{(2)}, \Phi_{2}^{(2)}\right]^{T}=\left[A_{n}, A_{u}, A_{\Phi}\right]^{T} n_{1}^{(1)^{2}}
$$


where,

$$
\begin{gathered}
A_{n}=\left(k^{2}+\alpha_{1}\right)\left[\frac{\tilde{\omega}^{2}}{k^{2}}\left(\frac{3}{2} \gamma_{1}-\frac{\tilde{\omega}}{k} \gamma_{2}\right)+\frac{3}{2} \sigma+A_{\Phi}\right], \\
A_{u}=\frac{\tilde{\omega}}{k}\left(A_{n}-1\right), \\
A_{\Phi}=\frac{\left(k^{2}+\alpha_{1}\right)}{3 k^{2}}\left[\frac{\tilde{\omega}^{2}}{k^{2}}\left(\frac{3}{2} \gamma_{1}-\frac{\tilde{\omega}}{k} \gamma_{2}\right)+\frac{3}{2} \sigma-\frac{\alpha_{2}}{\left(k^{2}+\alpha_{1}\right)^{3}}\right], \\
\tilde{\lambda}=\lambda-u_{0},
\end{gathered}
$$

and

$$
\gamma_{2}=\frac{3 u_{0}}{2 C^{2}}
$$

The second-order quantities with zeroth harmonic are determined from $l=0$ components of third order $\mathrm{O}\left(\varepsilon^{3}\right)$ and are given by,

$$
\left[n_{2}^{(0)}, u_{2}^{(0)}, \Phi_{2}^{(0)}\right]^{T}=\left[B_{n}, B_{u}, B_{\Phi}\right]^{T}\left|n_{1}^{(1)}\right|^{2}
$$

where

$$
\begin{gathered}
B_{n}=\frac{1}{\tilde{\lambda}}\left[\frac{2 \tilde{\omega}}{k}+B_{u}\right], \\
B_{u}=\frac{1}{z}\left[\frac{\tilde{\omega}^{2} \tilde{\lambda}^{2}}{k^{2}}\left(\frac{\gamma_{1}}{\tilde{\lambda}}-2 \gamma_{2}\right)+3 \sigma\left(\tilde{\lambda}+\frac{2 \tilde{\omega}}{k}\right)+\frac{2 \tilde{\omega}}{\alpha_{1} k}-\frac{2 \alpha_{2} \tilde{\lambda}}{\alpha_{1}\left(k^{2}+\alpha_{1}\right)^{2}}\right], \\
B_{\Phi}=\frac{1}{\alpha_{1}}\left[\frac{B_{n}\left(k^{2}+\alpha_{1}\right)^{2}-2 \alpha_{2}}{\left(k^{2}+\alpha_{1}\right)^{2}}\right],
\end{gathered}
$$

and

$$
z=\gamma_{1} \tilde{\lambda}^{2}-3 \sigma-\frac{1}{\alpha_{1}}
$$

Finally, we obtain the NST equation from $\mathrm{O}\left(\varepsilon^{3}\right)$ for $l=1$ components by using the above derived equations as,

$$
i \frac{\partial n_{1}^{(1)}}{\partial \tau}+P \frac{\partial^{2} n_{1}^{(1)}}{\partial \xi^{2}}+Q n_{1}^{(1)}\left|n_{1}^{(1)}\right|^{2}=0
$$

where

$$
P=\frac{-k^{2}}{2 \tilde{\omega} \gamma_{1}\left(k^{2}+\alpha_{1}\right)^{3}}\left[-\left(k^{2}-3 \alpha_{1}\right)+\frac{\left(k^{2}+\alpha_{1}\right)^{3}}{k^{2}}\left(\frac{\tilde{\omega}^{2} \gamma_{1}}{k^{2}}-\frac{2 \tilde{\omega} \gamma_{1} \tilde{\lambda}}{k}+\gamma_{1} \tilde{\lambda}^{2}\right)\right]=\frac{1}{2} \frac{\partial^{2} \tilde{\omega}}{\partial k^{2}}
$$

and

$$
Q=\frac{-k^{2}}{2 \tilde{\omega} \gamma_{1}}\left\{\begin{array}{l}
\left(\frac{\tilde{\omega}^{2} \gamma_{1}}{k^{2}}+3 \sigma\right)\left(A_{n}+B_{n}\right)+\frac{2 \tilde{\omega}}{k}\left(\gamma_{1}-\frac{\tilde{\omega}}{k} \gamma_{2}\right)\left(A_{u}+B_{u}\right) \\
-\frac{2 \alpha_{2}}{\left(k^{2}+\alpha_{1}\right)^{2}}\left(A_{\Phi}+B_{\Phi}\right)+2\left(\frac{\tilde{\omega}}{k}\right)^{3} \gamma_{2}-\frac{3}{2 C^{2}}\left(\frac{\tilde{\omega}}{k}\right)^{4}-\frac{3 \alpha_{3}}{\left(k^{2}+\alpha_{1}\right)^{4}}
\end{array}\right\} .
$$

Equation (15) satisfies the evolution of the complex amplitude of the nonlinear ion acoustic waves (IAWs) propagating in a weakly relativistic warm with nonextensively electrons on the basis of the fluid model in the finite wavenumber region.

For small wavenumber, equation (15) reduces to (appendix)

$$
i \frac{\partial n_{1}^{(1)}}{\partial \tau}-\frac{3}{2} \frac{b k}{\alpha_{1}^{2}} \frac{\partial^{2} n_{1}^{(1)}}{\partial \xi^{2}}+\frac{1}{3 k} \frac{a^{2} \alpha_{1}^{2}}{b} n_{1}^{(1)}\left|n_{1}^{(1)}\right|^{2}=0
$$


where

$$
a=\left[\frac{\gamma_{1}\left(3 \sigma \alpha_{1}+1\right)}{\alpha_{1}}\right]^{\frac{-1}{2}}\left\{\frac{\left(3 \sigma \alpha_{1}+1\right)\left(3 \alpha_{1}^{2}-2 \alpha_{2}\right)}{2 \alpha_{1}^{3}}+\frac{3}{2} \frac{\sigma\left(2 \alpha_{2}+\alpha_{1}^{2}\right)}{\alpha_{1}^{2}}-\frac{\gamma_{2}}{\gamma_{1}^{3 / 2}}\left(\frac{3 \sigma \alpha_{1}+1}{\alpha_{1}}\right)^{\frac{3}{2}}\right\}
$$

and

$$
b=\left[\frac{\gamma_{1}\left(3 \sigma \alpha_{1}+1\right)}{\alpha_{1}}\right]^{\frac{-1}{2}}
$$

\section{DERIVATION K-DV EQUATION FOR THE SYSTEM}

If we apply the reductive perturbation theory, we can show that the amplitude of the perturbed ion density in a weakly relativistic warm plasma and nonextensively distributed electrons in the small wavenumber limit is governed by the K-dV equation. This equation can be derived by introducing the stretched variables $\tau$ and $\xi$ as [30,31]

$$
\xi=\mu^{\frac{1}{2}}(x-\bar{\lambda} t) \text { and } \tau=\mu^{\frac{3}{2}} t .
$$

Thus,

$$
\left.\begin{array}{r}
\frac{\partial}{\partial x}=\mu^{\frac{1}{2}} \frac{\partial}{\partial \xi}, \\
\frac{\partial}{\partial t}=\mu^{\frac{1}{2}}\left(-\bar{\lambda} \frac{\partial}{\partial \xi}+\mu \frac{\partial}{\partial \tau}\right),
\end{array}\right\}
$$

and we expand the dependent variables as[29],

$$
\left.\begin{array}{r}
n=1+\mu \tilde{n}_{1}+\mu^{2} \tilde{n}_{2}+\mu^{3} \tilde{n}_{3}+\ldots \\
u=u_{0}+\mu u_{1}+\mu^{2} u_{2}+\mu^{3} u_{3}+\ldots \\
\Phi=\mu \Phi_{1}+\mu^{2} \Phi_{2}+\mu^{3} \Phi_{3}+\ldots
\end{array}\right\}
$$

where $\tilde{n}$ is the perturbed ion density and $\mu$ is the ordering parameter and is a measure of the size of the wavenumber $\mathrm{k}$; that is, $\mathrm{k}=O\left(\mu^{1 / 2}\right)$. Using Eqs. (17) in basic set of Eqs. (1)-(3) and equating the similar power coefficients, the lowest order terms of Iij are written as

$$
\left.\begin{array}{c}
\tilde{n}=\alpha_{1} \Phi_{1}, \\
u_{1}=\grave{\lambda} \tilde{n}_{1}, \\
\text { and } \\
\Phi_{1}=\frac{\tilde{n}_{1}}{\alpha_{1}},
\end{array}\right\}
$$

where

$$
\grave{\lambda}=\bar{\lambda}-u_{0}
$$

Poisson's equation gives the compatibility condition

$$
\left(\grave{\lambda}^{2} \gamma_{1}-3 \sigma\right) \alpha_{1}=1
$$

The next order of $\mu$ gives,

$$
\frac{\partial \tilde{n}_{1}}{\partial \tau}-\left(\bar{\lambda}-u_{0}\right) \frac{\partial \tilde{n}_{2}}{\partial \xi}+\frac{\partial u_{2}}{\partial \xi}+\grave{\lambda} \frac{\partial \tilde{n}_{1}^{2}}{\partial \xi}=0
$$




$$
\gamma_{1} \grave{\lambda} \frac{\partial \tilde{n}_{1}}{\partial \tau}-\left(\bar{\lambda}-u_{0}\right) \frac{\partial}{\partial \xi}\left(\gamma_{1} u_{2}+\gamma_{2} \grave{\lambda}^{2} \tilde{n}_{1}^{2}\right)+\gamma_{1} \grave{\lambda}^{2} \tilde{n}_{1} \frac{\partial \tilde{n}_{1}}{\partial \xi}+3 \sigma\left[\frac{\partial \tilde{n}_{2}}{\partial \xi}+\tilde{n}_{1} \frac{\partial \tilde{n}_{1}}{\partial \xi}\right]+\frac{\partial \Phi_{2}}{\partial \xi}=0
$$

and

$$
\frac{\partial^{2} \Phi_{1}}{\partial \xi^{2}}=\alpha_{1} \Phi_{2}+\alpha_{2} \frac{\tilde{n}_{1}^{2}}{\alpha_{1}^{2}}-\tilde{n}_{2}
$$

Eliminating the second order perturbed quantities and using the results of the pervious order with some algebraic manipulations we obtain the K-dV equation, which describes the evolution of the nonlinear ion acoustic waves,

$$
\frac{\partial \tilde{n}}{\partial \tau}+a \tilde{n} \frac{\partial \tilde{n}}{\partial \xi}+\frac{b}{2 \alpha_{1}^{2}} \frac{\partial^{3} \tilde{n}}{\partial \xi^{3}}=0
$$

where $\mathrm{a}$ and $\mathrm{b}$ are written as

$$
a=\left[\frac{\gamma_{1}\left(3 \sigma \alpha_{1}+1\right)}{\alpha_{1}}\right]^{\frac{-1}{2}}\left\{\frac{\left(3 \sigma \alpha_{1}+1\right)\left(3 \alpha_{1}^{2}-2 \alpha_{2}\right)}{2 \alpha_{1}^{3}}+\frac{3}{2} \frac{\sigma\left(2 \alpha_{2}+\alpha_{1}^{2}\right)}{\alpha_{1}^{2}}-\frac{\gamma_{2}}{\gamma_{1}^{3 / 2}}\left(\frac{3 \sigma \alpha_{1}+1}{\alpha_{1}}\right)^{\frac{3}{2}}\right\}
$$

and

$$
b=\left[\frac{\gamma_{1}\left(3 \sigma \alpha_{1}+1\right)}{\alpha_{1}}\right]^{\frac{-1}{2}}
$$

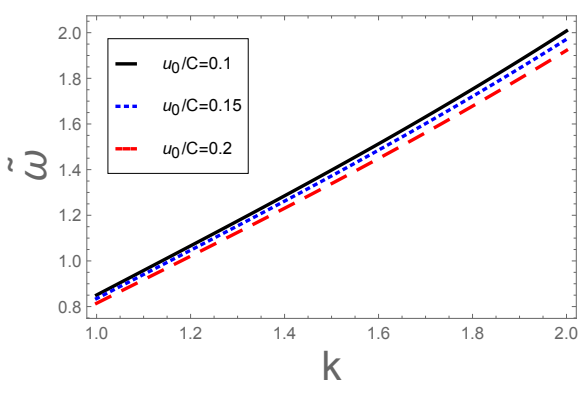

(a)

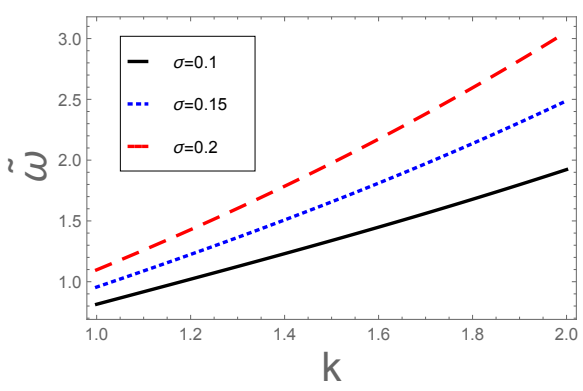

(b)

Figure 1: The variation of the angular frequency $(\tilde{\omega})$ with wavenumber $(\mathrm{k})$ : (a) for different values of $u_{0} / C$ and $\sigma=0.1$, (b) for different values of $\sigma$ and $u_{0} / C=0.2$. Here the nonextensive parameter $q=0.55$.

\section{DERIVATION OF NST EQUATION FROM K-DV EQUATION (SMALL WAVENUMBER APPROXIMATION)}

To obtain the NST equation from K-dV equation (20) we follow the work by recently by El-Labany et al.[24] and we reach at

$$
i \frac{\partial \tilde{n}_{1}^{(1)}}{\partial \sigma}-\frac{3}{2} \frac{b k}{\alpha_{1}^{2}} \frac{\partial^{2} \tilde{n}_{1}^{(1)}}{\partial \chi^{2}}+\frac{1}{3 k} \frac{a^{2} \alpha_{1}^{2}}{b} \tilde{n}_{1}^{(1)}\left|\tilde{n}_{1}^{(1)}\right|^{2}=0 .
$$

which similar with the small wavenumber limit of equation (15) i.e. equation (16). 

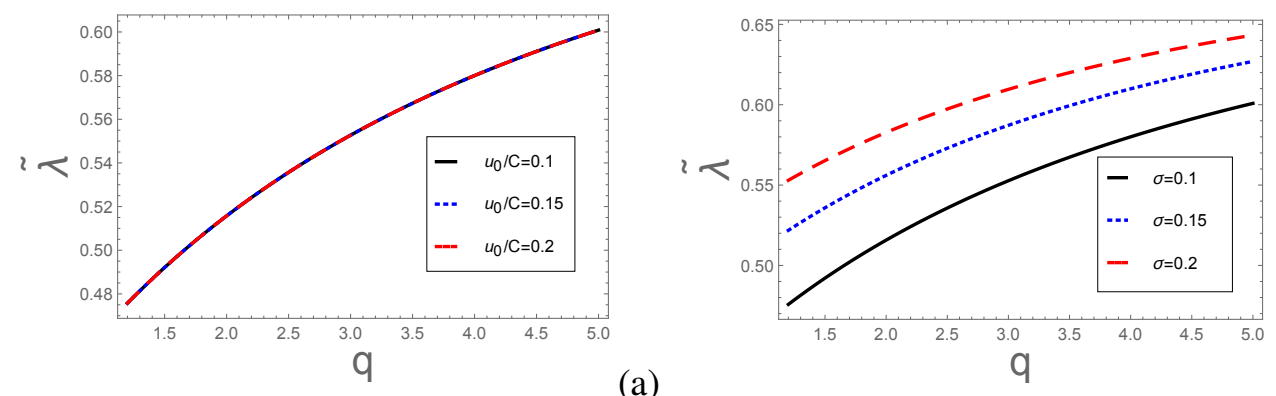

(a)

(b)

Figure 2: The variation of the group velocity $(\tilde{\lambda})$ with nonextensive parameter q: (a) for different values of $u_{0} / C$ and $\sigma=0.1$, (b) for different values of $\sigma$ and $u_{0} / C=0.2$. Here $k=1.4$.

\section{RESULTS AND DISCUSSION}

Figures (1.a) and (1.b) show the numerical analysis of Eq. (9a) to examine the linear properties of the IAWs for different values of relativistic factor $\left(u_{0} / C\right)$, temperatures ratio $\sigma\left(=T_{i} / T_{e}\right)$ with the value of the nonextensive parameter $q=0.55$. These figures show that, the phase velocity decreases with increasing $u_{0} / C$ and is enhanced with increasing $\sigma$. Also, figures (2.a) and (2.b) show the group velocity properties for different values of $u_{0} / C$ and $\sigma$, which are given in Eq. (9b). The group velocity is independent on the variation of $u_{0} / C$ but varies with the nonextensive parameter q, and increases with increasing $\sigma$.

On the other hand, we investigated the variation of the critical wavenumbers (higher and lower wavenumber) with $u_{0} / C$ for different values of $\sigma(\sigma=0.1$ and $\sigma=0.2)$ as shown in figures (3.a) and (3.b). These figures show that the upper critical wavenumber decreases as $\sigma$ increases while the lower wavenumber remains constant, where $\sigma\left(=T_{i} / T_{e}\right)$ is always very low $\simeq 0.2$. Figures (4.a) and (4.b) show the variation of the critical wavenumbers with $\sigma$ for different values of the nonextensive parameter q. We notice that, the upper critical wavenumber increases with increasing the nonextensive parameter $\mathrm{q}$ and we have only one lower wavenumber which decreases as $\sigma$ increase.
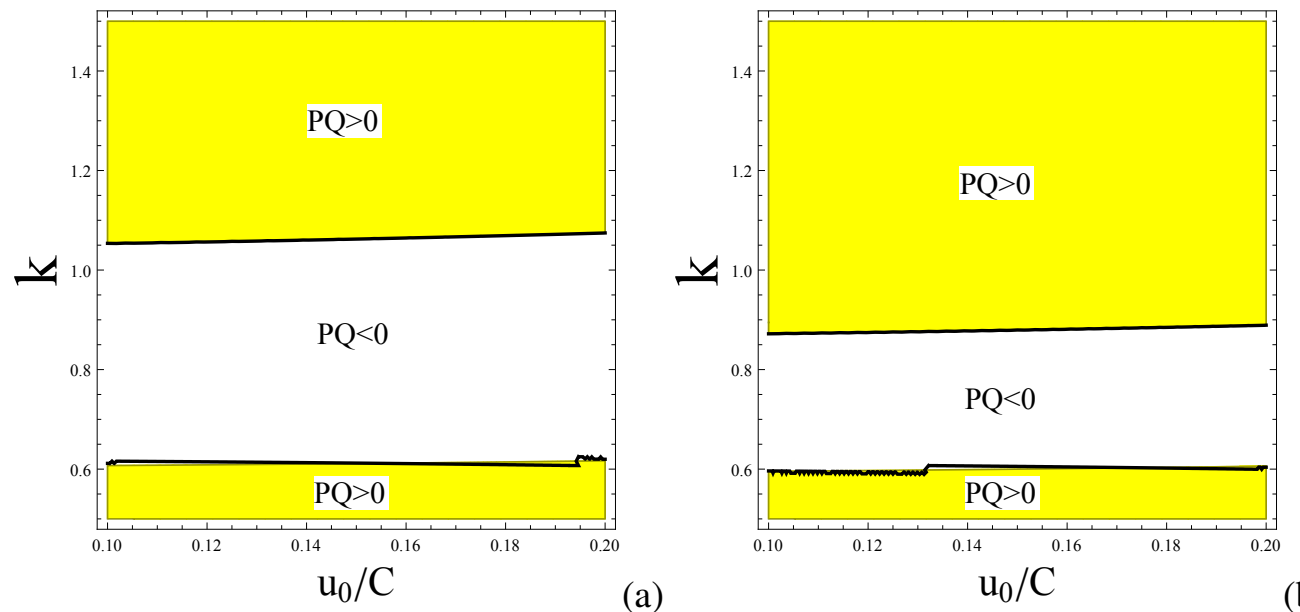

Figure 3: Contour plot of the product $P Q=0$, depicted against k and $u_{0} / C$ : (a) for $\sigma=0.1$, (b) for $\sigma=0.2$. Here the nonextensive parameter $q=0.55$, where the (white) yellow region represents the (stability) instability region. 

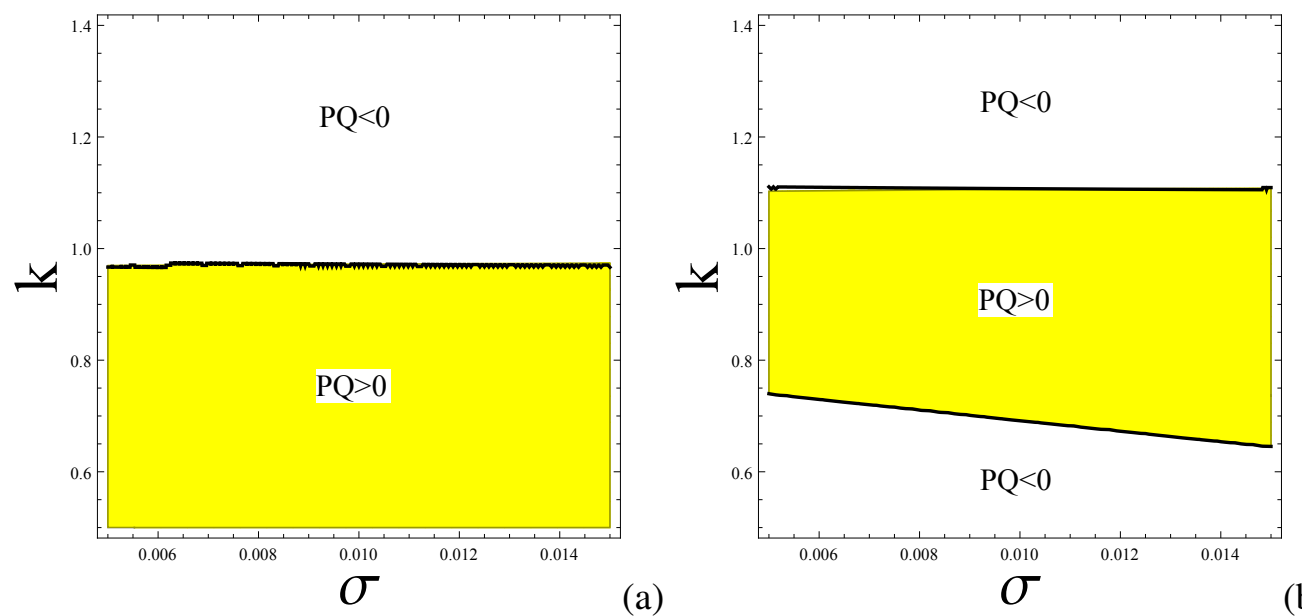

(b)

Figure 4: Contour plot of the product $P Q=0$, depicted against $\mathrm{k}$ and $\sigma$ : (a) for the nonextensive parameter $q=2.87$, (b) for the nonextensive parameter $q=4$. Here $u_{0} / C=0.2$, where the (white) yellow region represents the (stability) instability region.

Figure 5 shows that, the effect of the q-nonextensively parameter on the stability and instability domains. We find that the increases of the q parameter increase the lower and higher critical wavenumbers. Also this figure shows that when q parameter increase the stable region becomes more narrow. The stable region at $\sigma=0.1$ (figure (5.a)) is larger than the stable region at $\sigma=0.2$ (figure (5.b)) this mean that the system gains more energy and becomes more unstable when the ion temperature $T_{i}$ increases.
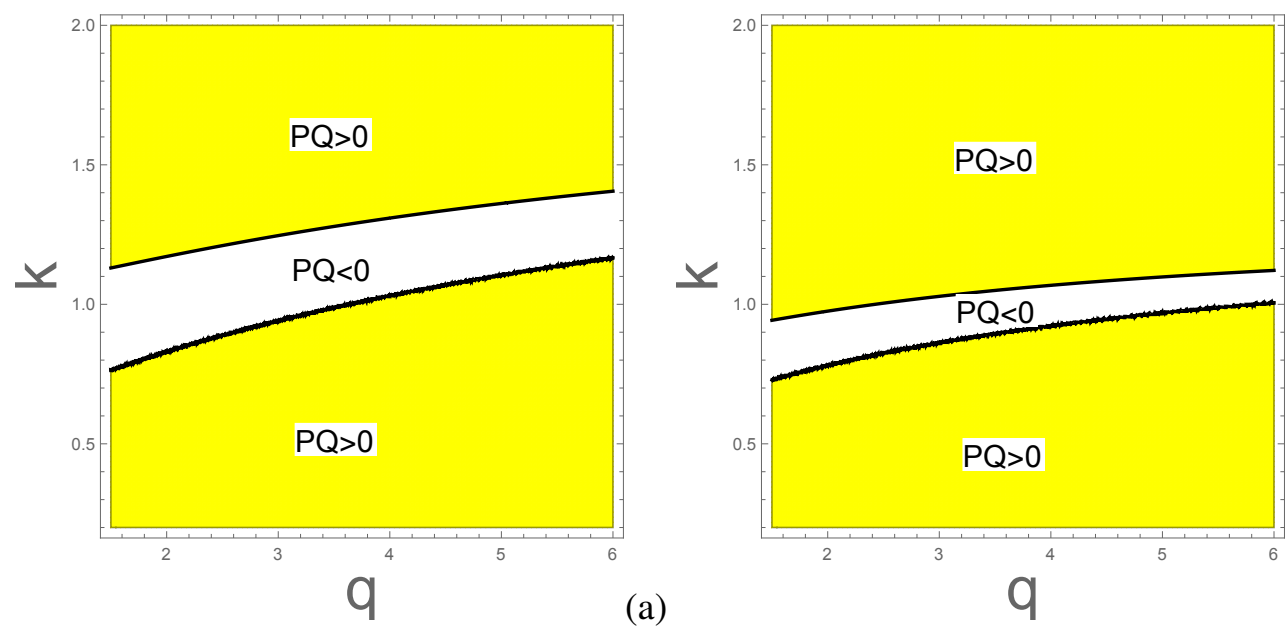

Figure 5: Contour plot of the product $P Q=0$, depicted against $\mathrm{k}$ and $q$ : (a) for $\sigma=0.1$, (b) for $\sigma=0.2$. Here $u_{0} / C=0.1$, where the (white) yellow region represents the (stability) instability region.

The comparison between the results obtained by nonextensively and Maxwellian (at the limit of $q \longrightarrow 1$ ) distributed electrons are displayed in figures (6.a) and (6.b). It is obvious from these figures that, the unstable region for nonextensively distributed electrons is larger than the unstable region for Maxwellian. This means that the nonextensive particles have high energy than the Maxwellian ones which increase the instability of the system. So, from these results we find that the Maxwellian distribution is inadequate for explain the highly energetic particles vice the nonextensive distribution. 

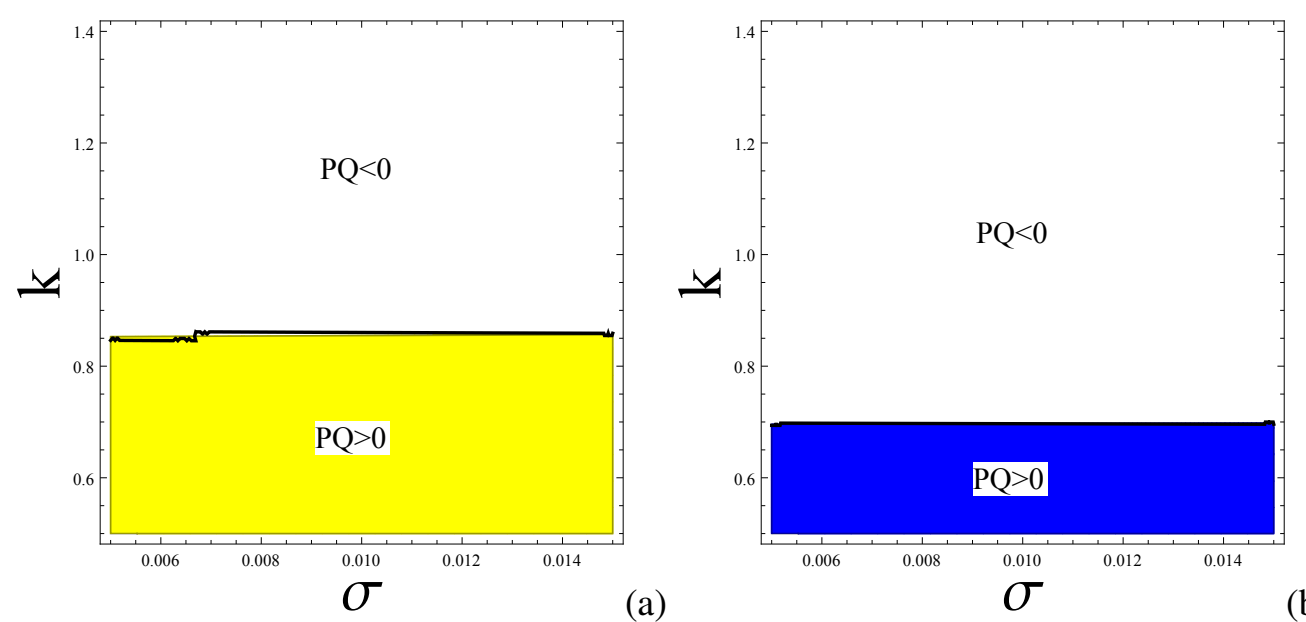

Figure 6: Comparison between the contour plot of the product $P Q=0$, depicted against k and $\sigma$ obtained by the nonextensively distributed electrons in (a) at the nonextensive parameter $q=2$ and the Maxwellian distributed electrons in (b), where the (white) blue region represents the (stability) instability region.

\section{CONCLUSION}

In the present work, we employed the nonlinear hydrodynamic equations of a weakly relativistic unmagnetized adiabatic plasma model including warm ions and nonextensively distributed electrons. This system of equations is reduced to the NST equation for finite wavenumber by using the multiple scales method in which the coefficients of this equation are strongly dependent on both ion temperature $\sigma$ and the ion streaming velocity $\left(\gamma_{1}, \gamma_{2}\right)$. We also found good agreement between the small-wavenumber limit of the NST equation obtained by using multiple scales method and the NST equation obtained from $\mathrm{K}-\mathrm{dV}$ equation obtained by using the reductive perturbation method. It is well known that the stability condition for the NST equation is $P Q<0$. Since $\mathrm{P}$ is always negative for $\omega>k u_{0}$, one has to determine the value of the critical wavenumber $k_{C}$ at which $\mathrm{Q}$ vanishes. Then, for all values of $k>k_{C}$, the wave has modulation instability, while modulation stability for all values of $k>k_{C}$. Moreover we investigated the dependence of the stability/instability regions on the physical parameters $\sigma, u_{0} / C$ and q characterizing the system. A comparison between nonextensivelly and Maxwellian distributed electrons is carried out and the validity of our results in astrophysical and finement fusion plasma. Finally, To show the validity of our results, we consider the cold nonrelativistic limit $\left(\sigma=0\right.$ and $\left.u_{0} / C=0\right)$ of the present work which is found to agree with the work done previously by Bains et al. [21].

A large number of observations clearly reveal the existence of a weakly relativistic warm plasma and nonextensively distributed electrons in astrophysics environments such as solar neutrino problem, stellar polytropes and galaxy cluster as well as finement fusion plasma [20, 21].

\section{Appendix}

To estimate the coefficients of the NST equation for small wavenumber (k), firstly we calculate the different terms appearing in these coefficients. 
From Eqs.(9), as $k \longrightarrow 0$, we have

$$
\frac{\tilde{\omega}}{k} \approx \tilde{\lambda} \approx\left[\frac{1+3 \sigma \alpha_{1}}{\gamma_{1} \alpha_{1}}\right]^{1 / 2}
$$

thus

$$
\left[\begin{array}{l}
1 \\
z
\end{array}\right]=\left[\frac{\alpha_{1}\left(k^{2}+\alpha_{1}\right)}{k^{2}}\left\{k^{2} \alpha_{1}\left(3 \gamma_{1} \sigma\left(k^{2}+\alpha_{1}\right)+1\right)-\left(k^{2}+3 \alpha_{1}\right)\left(k^{2}+\alpha_{1}\right)\right\}^{-1}\right]=\frac{-\alpha_{1}^{2}}{3 k^{2}}
$$

where the square-bracket notation indicates the quantity is estimated at small wave number $(k \longrightarrow 0)$.

Also

$$
\begin{aligned}
A_{n}=\left(k^{2}+\alpha_{1}\right) & \left\{\frac{\tilde{\omega}^{2}}{k^{2}}\left(\frac{3}{2} \gamma_{1}-\frac{\tilde{\omega}}{k} \gamma_{2}\right)+\frac{3}{2} \sigma+\frac{\left(k^{2}+\alpha_{1}\right)}{3 k^{2}}\left[\frac{\tilde{\omega}^{2}}{k^{2}}\left(\frac{3}{2} \gamma_{1}-\frac{\tilde{\omega}}{k} \gamma_{2}\right)+\frac{3}{2} \sigma-\frac{\alpha_{2}}{\left(k^{2}+\alpha_{1}\right)^{3}}\right]\right\} \\
& \approx \frac{\alpha_{1}^{2}}{3 k^{2}}\left[\frac{3}{2}\left(\frac{1+3 \sigma \alpha_{1}}{\alpha_{1}}\right)-\frac{\gamma_{2}}{\gamma_{1}^{3 / 2}}\left(\frac{1+3 \sigma \alpha_{1}}{\alpha_{1}}\right)^{3 / 2}+\frac{3}{2} \sigma-\frac{\alpha_{2}}{\alpha_{1}^{3}}\right],
\end{aligned}
$$

$$
\begin{aligned}
& A_{u}= \frac{\tilde{\omega}}{k}\left(A_{n}-1\right) \\
& \approx \frac{\alpha_{1}^{2}}{3 k^{2}}\left[\frac{1+3 \sigma \alpha_{1}}{\gamma_{1} \alpha_{1}}\right]^{1 / 2}\left[\frac{3}{2}\left(\frac{1+3 \sigma \alpha_{1}}{\alpha_{1}}\right)-\frac{\gamma_{2}}{\gamma_{1}^{3 / 2}}\left(\frac{1+3 \sigma \alpha_{1}}{\alpha_{1}}\right)^{3 / 2}+\frac{3}{2} \sigma-\frac{\alpha_{2}}{\alpha_{1}^{3}}\right], \\
& A_{\Phi}= \frac{\left(k^{2}+\alpha_{1}\right)}{3 k^{2}}\left[\frac{\tilde{\omega}^{2}}{k^{2}}\left(\frac{3}{2} \gamma_{1}-\frac{\tilde{\omega}}{k} \gamma_{2}\right)+\frac{3}{2} \sigma-\frac{\alpha_{2}}{\left(k^{2}+\alpha_{1}\right)^{3}}\right], \\
& \approx \frac{\alpha_{1}^{2}}{3 k^{2}}\left[\frac{3}{2}\left(\frac{1+3 \sigma \alpha_{1}}{\alpha_{1}}\right)-\frac{\gamma_{2}}{\gamma_{1}^{3 / 2}}\left(\frac{1+3 \sigma \alpha_{1}}{\alpha_{1}}\right)^{3 / 2}+\frac{3}{2} \sigma-\frac{\alpha_{2}}{\alpha_{1}^{3}}\right], \\
& B_{n}=\frac{1}{\tilde{\lambda}}\left\{\frac{2 \tilde{\omega}}{k}+\frac{1}{z}\left[\frac{\tilde{\omega}^{2} \tilde{\lambda}^{2}}{k^{2}}\left(\frac{\gamma_{1}}{\tilde{\lambda}}-2 \gamma_{2}\right)+3 \sigma\left(\tilde{\lambda}+\frac{2 \tilde{\omega}}{k}\right)+\frac{2 \tilde{\omega}}{\alpha_{1} k}-\frac{2 \alpha_{2} \tilde{\lambda}}{\alpha_{1}\left(k^{2}+\alpha_{1}\right)^{2}}\right]\right\} \\
& \approx-\frac{\alpha_{1}^{2}}{3 k^{2}}\left[\gamma_{1}\left(\frac{1+3 \sigma \alpha_{1}}{\gamma_{1} \alpha_{1}}\right)-2 \gamma_{2}\left(\frac{1+3 \sigma \alpha_{1}}{\gamma_{1} \alpha_{1}}\right)^{3 / 2}+9 \sigma+\frac{2}{\alpha_{1}}-\frac{2 \alpha_{2}}{\alpha_{1}^{3}}\right], \\
& B_{u}=\frac{1}{z}\left[\frac{\tilde{\omega}^{2} \tilde{\lambda}^{2}}{k^{2}}\left(\frac{\gamma_{1}}{\tilde{\lambda}}-2 \gamma_{2}\right)+3 \sigma\left(\tilde{\lambda}+\frac{2 \tilde{\omega}}{k}\right)+\frac{2 \tilde{\omega}}{\alpha_{1} k}-\frac{2 \alpha_{2} \tilde{\lambda}}{\alpha_{1}\left(k^{2}+\alpha_{1}\right)^{2}}\right] \\
& \approx-\frac{\alpha_{1}^{2}}{3 k^{2}}\left(\frac{1+3 \sigma \alpha_{1}}{\gamma_{1} \alpha_{1}}\right)^{1 / 2}\left[\gamma_{1}\left(\frac{1+3 \sigma \alpha_{1}}{\gamma_{1} \alpha_{1}}\right)-2 \gamma_{2}\left(\frac{1+3 \sigma \alpha_{1}}{\gamma_{1} \alpha_{1}}\right)^{3 / 2}+9 \sigma+\frac{2}{\alpha_{1}}-\frac{2 \alpha_{2}}{\alpha_{1}^{3}}\right],
\end{aligned}
$$

and

$$
\begin{aligned}
B_{\Phi}=\frac{1}{\alpha_{1}} & {\left[\frac{B_{n}\left(k^{2}+\alpha_{1}\right)^{2}-2 \alpha_{2}}{\left(k^{2}+\alpha_{1}\right)^{2}}\right] } \\
& \approx-\frac{\alpha_{1}}{3 k^{2}}\left[\gamma_{1}\left(\frac{1+3 \sigma \alpha_{1}}{\gamma_{1} \alpha_{1}}\right)-2 \gamma_{2}\left(\frac{1+3 \sigma \alpha_{1}}{\gamma_{1} \alpha_{1}}\right)^{3 / 2}+9 \sigma+\frac{2}{\alpha_{1}}-\frac{2 \alpha_{2}}{\alpha_{1}^{3}}\right],
\end{aligned}
$$


Thus the coefficients $\mathrm{P}$ and $\mathrm{Q}$ are given by

$$
\begin{aligned}
P= & \frac{-k^{2}}{2 \tilde{\omega} \gamma_{1}\left(k^{2}+\alpha_{1}\right)^{3}}\left[-\left(k^{2}-3 \alpha_{1}\right)+\frac{\left(k^{2}+\alpha_{1}\right)^{3}}{k^{2}}\left(\frac{\tilde{\omega}^{2} \gamma_{1}}{k^{2}}-\frac{2 \tilde{\omega} \gamma_{1} \tilde{\lambda}}{k}+\gamma_{1} \tilde{\lambda}^{2}\right)\right] \\
& \approx-\frac{1}{2} \frac{k}{\gamma_{1} \alpha_{1}^{3}}\left[\frac{1+3 \sigma \alpha_{1}}{\gamma_{1} \alpha_{1}}\right]^{-1 / 2}\left[3 \alpha_{1}\right]=-\frac{3}{2} \frac{k}{\alpha_{1}^{2}}\left[\frac{1+3 \sigma \alpha_{1}}{\gamma_{1} \alpha_{1}}\right]^{-1 / 2}=-\frac{3}{2} \frac{b}{\alpha_{1}^{2}} k
\end{aligned}
$$

and

$$
\begin{gathered}
Q=\frac{-k^{2}}{2 \tilde{\omega} \gamma_{1}}\left\{\begin{array}{c}
\left(\frac{\tilde{\omega}^{2} \gamma_{1}}{k^{2}}+3 \sigma\right)\left(A_{n}+B_{n}\right)+\frac{2 \tilde{\omega}}{k}\left(\gamma_{1}-\frac{\tilde{\omega}}{k} \gamma_{2}\right)\left(A_{u}+B_{u}\right) \\
-\frac{2 \alpha_{2}}{\left(k^{2}+\alpha_{1}\right)^{2}}\left(A_{\Phi}+B_{\Phi}\right)+2\left(\frac{\tilde{\omega}}{k}\right)^{3} \gamma_{2}-\frac{3}{2 C^{2}}\left(\frac{\tilde{\omega}}{k}\right)^{4}-\frac{3 \alpha_{3}}{\left(k^{2}+\alpha_{1}\right)^{4}}
\end{array}\right\} \\
\approx \frac{\alpha_{1}^{2}}{3 k}\left[\frac{\gamma_{1}\left(1+3 \sigma \alpha_{1}\right)}{\alpha_{1}}\right]^{-1 / 2}\left[\frac{\left(3 \sigma \alpha_{1}+1\right)\left(3 \alpha_{1}^{2}-2 \alpha_{2}\right)}{2 \alpha_{1}^{3}}+\frac{3}{2} \frac{\sigma\left(2 \alpha_{2}+\alpha_{1}^{2}\right)}{\alpha_{1}^{2}}-\frac{\gamma_{2}}{\gamma_{1}^{\frac{3}{2}}}\left(\frac{\gamma_{1}\left(1+3 \sigma \alpha_{1}\right)}{\alpha_{1}}\right)^{\frac{3}{2}}\right] \\
=\frac{1}{3 k} \frac{a^{2} \alpha_{1}^{2}}{b}
\end{gathered}
$$

\section{REFERENCES}

[1] Takuji Kawahara. The derivative-expansion method and nonlinear dispersive waves. Journal of the Physical Society of Japan, 35(5):1537-1544, 1973.

[2] Yasunori Nejoh. The effect of the ion temperature on the ion acoustic solitary waves in a collisionless relativistic plasma. Journal of Plasma Physics, 37(3):487âĂŞ495, 1987.

[3] S. Sultana and I. Kourakis. Electrostatic solitary waves in the presence of excess superthermal electrons: modulational instability and envelope soliton modes. Plasma Physics and Controlled Fusion, 53(4):045003, April 2011.

[4] S. V. Vladimirov, V. N. Tsytovich, S. I. Popel, and F. KH Khakimov. Modulational Interactions in Plasmas. (Kluwer Academic, Dordrecht). 1995.

[5] A. A. Vedenov and L. I Rudakov. Theory of Turbulent Sov, Phys. Doklady 9 1073. 1965.

[6] H Ikezi, RJ Taylor, and DR Baker. Formation and interaction of ion-acoustic solitions. Physical Review Letters, 25(1):11, 1970.

[7] S Watanabe. Self-modulation of a nonlinear ion wave packet. Journal of Plasma Physics, 17(3):487-501, 1977.

[8] Xue Jukui and Lang He. Modulational instability of cylindrical and spherical dust ion-acoustic waves. Physics of Plasmas, 10(2):339-342, 2003.

[9] AlfrÃl'd Renyi. On a new axiomatic theory of probability. 6:285, 1955.

[10] Constantino Tsallis. Possible generalization of boltzmann-gibbs statistics. Journal of Statistical Physics, 52(1/2), 1988. 
[11] F Sattin. Non-extensive entropy from incomplete knowledge of shannon entropy? Physica Scripta, 71.5:443, 2005.

[12] VÃ Nonlinear Processes in Geophysics, 13:237âĂŞ241, 2006.

[13] M. P. Leubner. Fluctuation in nonextensive reactionâĂŞdiffusion systems. Nonlinear Processes in Geophysics, 75.5:722, 2007.

[14] Junlin Wu and Huaijun Chen. Consequences of entropy bifurcation in non-maxwellian astrophysical environments. Nonlinear Processes in Geophysics, 15.4:531, 2008.

[15] Rudolf Hanel and Stefan Thurner. Stability criteria for q-expectation values. Physics Letters A 373, 16:1415-1420, 2009.

[16] Rabia Amour and Mouloud Tribecher. Variable charge dust acoustic solitary waves in a dusty plasma with aq-nonextensive electron velocity distribution. Physics of Plasmas, 17.6:063702, 2010.

[17] Mouloud Tribeche, Lyes Djebarni, and Rabia Amour. Ion-acoustic solitary waves in a plasma with aq-nonextensive electron velocity distribution. Physics of Plasmas, 17(4):042114, 2010.

[18] M Amina, S A Ema, and A A Mamun. Nonplanar electrostatic shock waves in an opposite polarity dust plasma with nonextensive electrons and ions. Pramana âAŞ J. Phys, 88:532-546, 2017.

[19] Demiray, Hilmi, E. R. El-Zahar, and S. A. Shan. On progressive wave solution for non-planar kdv equation in a plasma with q-nonextensive electrons and two oppositely charged ions. TWMS J. APP. AND ENG. MATH, 10(2):0973-7111, 2020.

[20] Hamid Reza Pakzad. Ion acoustic solitary waves in a weakly relativistic plasma with qnonextensive electrons and thermal positrons. Astrophysics and Space Science, 334(2):337-343, 2011.

[21] AS Bains, Mouloud Tribeche, and TS Gill. Modulational instability of ion-acoustic waves in a plasma with a q-nonextensive electron velocity distribution. Physics of Plasmas, 18(2):022108, 2011.

[22] SK El-Labany. Weakly relativistic effect on the modulation of nonlinear ion-acoustic waves in a warm plasma. Journal of plasma physics, 54(3):295-308, 1995.

[23] SK El-Labany, MS Abdel Krim, SA El-Warraki, and WF El-Taibany. Modulational instability of a weakly relativistic ion acoustic wave in a warm plasma with nonthermal electrons. Chinese Physics, 12(7):759, 2003.

[24] Salah K El-Labany, Wael F El-Taibany, Nabila A El-Bedwehy, and Nora A El-Shafeay. Modulation of the nonlinear ion acoustic waves in a weakly relativistic warm plasma with superthermally distributed electrons. Alfarama Journal of Basic \& Applied Sciences, 2020.

[25] Jie-fang Zhang, Yue-yue Wang, and Lei Wu. Modulation instability of ion acoustic waves, solitons, and their interactions in nonthermal electron-positron-ion plasmas. Physics of Plasmas, 16(6):062102, 2009. 
[26] Shimin Guo and Liquan Mei. Modulation instability and dissipative rogue waves in ion-beam plasma: Roles of ionization, recombination, and electron attachment. Physics of Plasmas, 21(11):112303, 2014.

[27] NA Chowdhury, A Mannan, MM Hasan, and AA Mamun. Modulational instability, ion-acoustic envelope solitons, and rogue waves in four-component plasmas. Plasma Physics Reports, 45(5):459-465, 2019.

[28] NA El-Bedwehy and Wael Farouk El-Taibany. Modulational instability of dust-ion acoustic waves in the presence of generalized (r, q) distributed electrons. Physics of Plasmas, 27(1):012107, 2020.

[29] Tarsem Singh Gill, Amandeep Singh, Harvinder Kaur, Nareshpal Singh Saini, and Parveen Bala. Ion-acoustic solitons in weakly relativistic plasma containing electron-positron and ion. Physics Letters A, 361(4-5):364-367, 2007.

[30] M Mehdipoor. K-dv and mk-dv equations for solitary waves in negative ion plasmas with nonmaxwellian electrons. Astrophysics and Space Science, 348(1):115-121, 2013.

[31] MM Rahman, MS Alam, and AA Mamun. Positron-acoustic gardner solitons and double layers in electron-positron-ion plasmas with nonthermal electrons and positrons. The European Physical Journal Plus, 129(5):84, 2014. 